\title{
BMJ Open Patient safety during radiological examinations: a nationwide survey of residency training hospitals in Taiwan
}

\author{
Yuan-Hao Lee, ${ }^{1}$ Clayton Chi-Chang Chen, ${ }^{2}$ San-Kan Lee, ${ }^{2}$ Cheng-Yu Chen, ${ }^{3,4}$ \\ Yung-Liang Wan, ${ }^{5}$ Wan-Yuo Guo, ${ }^{6}$ Amy Cheng, ${ }^{1}$ Wing P Chan ${ }^{1,4}$
}

To cite: Lee Y-H, Chen CC-C, Lee S-K, et al. Patient safety during radiological examinations: a nationwide survey of residency training hospitals in Taiwan. BMJ Open 2016;6: 010756 . doi:10.1136/bmjopen-2015010756

- Prepublication history and additional material is available. To view please visit the journal (http://dx.doi.org/ 10.1136/bmjopen-2015010756).

Received 8 December 2015 Revised 21 June 2016 Accepted 2 September 2016

CrossMark

For numbered affiliations see end of article.

Correspondence to Professor Wing P Chan; wingchan@tmu.edu.tw

\section{ABSTRACT}

Objectives: Variations in radiological examination procedures and patient load lead to variations in standards of care related to patient safety and healthcare quality. To understand the status of safety measures to protect patients undergoing radiological examinations at residency training hospitals in Taiwan, a follow-up survey evaluating the full spectrum of diagnostic radiology procedures was conducted.

Design: Questionnaires covering 12 patient safetyrelated themes throughout the examination procedures were mailed to the departments of diagnostic radiology with residency training programmes in 19 medical centres (with $>500$ beds) and 17 smaller local institutions in Taiwan. After receiving the responses, all themes in 2014 were compared between medical centres and local institutions by using $\chi^{2}$ or 2 -sample t-tests.

Participants: Radiology Directors or Technology Chiefs of medical centres and local institutions in Taiwan participated in this survey by completing and returning the questionnaires.

Results: The response rates of medical centres and local institutions were $95 \%$ and $100 \%$, respectively. As indicated, large medical centres carried out more frequent clinically ordered, radiologist-guided patient education to prepare patients for specific examinations (CT, $28 \%$ vs $6 \%$; special procedures, $78 \%$ vs $44 \%$ ) and incident review and analysis (89\% vs $47 \%$ ); however, they required significantly longer access time for MRI examinations $(7.00 \pm 29.50$ vs $3.50 \pm 3.50$ days $)$, had more yearly incidents of large-volume contrastmedium extravasation $(2.75 \pm 1.00$ vs $1.00 \pm 0.75$ cases $)$ and blank radiographs ( $41 \%$ vs $8 \%)$, lower monthly rates of suboptimal (but interpretable) radiographs $(0.00 \pm 0.01 \%$ vs $0.64 \pm 1.84 \%)$ and high-risk reminder reporting $(0.01 \pm 0.16 \%$ vs $1.00 \pm 1.75 \%)$ than local institutions.

Conclusions: Our study elucidates the status of patient safety in diagnostic radiology in Taiwan, thereby providing helpful information to improve patient safety guidelines needed for medical imaging in the future.

\section{INTRODUCTION}

In recent years, patient safety has garnered significant attention worldwide. In particular,

\section{Strengths and limitations of this study}

This study examines different patient safety and safety-related care standards for all phases of radiological examinations, including the preexamination, midexamination and postexamination phases.

- By comparing the status of patient safety in the existing clinical environments of diagnostic radiology, this study may provide important data to help decision makers improve radiological safety guidelines and procedures.

- The survey results have not yet been supported by data from site visits to the participating hospitals.

- The small number of participants as well as the discontinuity of their responses to this survey could potentially result in large SDs and a measurement bias.

several large epidemiological studies have revealed the large number of errors made or the high level of risk due to mistakes in current medical environments. ${ }^{1-3}$ Improving patient safety is paramount in all healthcare procedures and services. A recent disclosure of medical malpractice lawsuits involving MRI indicates that the sources of risk can be lack of clinical training of new recruits and insufficiency of accurate communication between the clinical team and MRI staff. ${ }^{4}$ To prevent malpractice, National Patient Safety Committees/Agencies worldwide have been established to develop policies that address certain safety issues as well as promote and support efforts to consolidate patient safety guidelines. ${ }^{5}{ }^{6}$ Thus far, there has been no specific operational guideline in Taiwan other than those dealing with instrumental quality assurance and dose minimisation for patient safety during the conduct of radiological examinations. ${ }^{7-9}$

According to the American Institute in Taiwan, differences in medical care between the West and Taiwan mainly arise from the 
large volume of patients that doctors attend to every day. ${ }^{10}$ This comparatively heavier workload in Taiwan reduces the time doctors spend with patients. Hence, it has become increasingly important to avoid any unintentional and unexpected outcomes during healthcare service delivery. In 2003, the Executive Yuan of Taiwan appointed a Patient Safety Committee to construct and enact guidelines for patient safety. The primary goal of healthcare services is patient safety; continuous medical education and adequate monitoring in healthcare settings would likely reduce medical malpractice and errors while improving safety in the healthcare environment. ${ }^{11}$ Ten patient-centred safety action plans were developed through the Conference of National Health Medical Policy. Plans like 'Establishment of medical malpractice reporting systems aimed at learning and making improvements', 'Stressing the importance of patient rights and strengthening the communication between patients and medical staff', 'Creating a safe and secure mechanism for drug administration' and 'Improving measures of intrahospital infection control' relate to quality and safety issues encountered in radiology such as occurrences of high-risk incidents during examinations or diagnostic procedures, patient understanding and consent for examination procedures, near-miss and missed cases, and side effects from intravenous contrast injection and interventional procedures.

In Taiwan, residency training hospitals can be categorised as medical centres and local and district hospitals. Local institutions have lower accreditation requirements such as a lower number of hospital beds and a lower level of emergency care than medical centres. ${ }^{12}$ Medical centres have $>500$ open acute beds and provide the services of around 25 different specialties (including Radiology, Radiation Oncology and Nuclear Medicine) accredited by the Ministry of Health and Welfare in Taiwan. It is estimated that every 7 of 10 patients visiting hospitals undergo radiological examinations. ${ }^{13}$ Because of this high rate, it is crucial to evaluate differences in the quality of diagnostic radiology services and the safety of examination procedures between highly accredited medical centres and other healthcare organisations that provide residency training programmes.

It is known that medical centres meet stricter accreditation standards than local and district hospitals in Taiwan. With differences in standards of care and radiological practices, the quality of patient safety at medical centres is expected to be different from that at local institutions. To gain insights into improvement initiatives in patient safety during radiological examinations, this cross-sectional, nationwide survey aimed to compare patient safety assessment in the departments of diagnostic radiology between medical centres and local institutions in Taiwan.

\section{METHODS}

This survey was conducted by mailing questionnaires to 19 medical centres and 17 local institutions in Taiwan.
The questionnaires were completed by Radiology Directors or the Technology Chiefs. Following the radiological examination procedures and safety metrics suggested by Johnson et al, ${ }^{14}$ this study assessed the status of patient safety in 2014 and the radiological errors in the three phases of examinations: the pre-examination, midexamination and postexamination phases. ${ }^{13} 15$

\section{Measures}

The pre-examination phase survey included topics in association with appropriateness of examination orders and practice, access and wait time for radiological examinations, healthcare education and medical order-based selection of imaging protocols. The midexamination phase survey covered issues of patient safety such as patient verification, prevention of patient falls, time-out procedures, side effects arising from intravenous contrast-medium administration, assessment of image quality and patient satisfaction. Finally, the postexamination phase safety survey focused on correction of examination results; reporting effectiveness of radiological examinations; high-risk reminder systems; and incidents, events and review conferences.

\section{Evaluation of the pre-examination phase}

Appropriateness of examination orders and practice was analysed by questioning whether radiologists had reviewed and approved the orders prior to the procedure being performed by radiologic technologists and whether additional information indicating appropriateness, such as clinical history, was provided with the examination order. The access times for examinations of CT, MRI, mammography, ultrasonography and special procedures (eg, angiography, lower and upper gastrointestinal radiography) were defined as the average wait times until the scheduled examinations. An investigation of healthcare education prior to examinations through flyers or oral presentation was carried out to find out whether patients received the required medical information from physicians or/and radiologic technologists and nurses. The use of medical order-based imaging protocols with preset imaging parameters or conditions of CT, MRI, mammography, ultrasonography and special procedures was studied.

\section{Evaluation of the midexamination phase}

Verification of patients' ID (using dual recognition methods, eg, involving name and date of birth) and pregnancy status as the first monitored checkpoint of an examination were surveyed as well as the circumstances of patient falls occurring during an examination. Respondents were also asked about the practice of taking 'time-out' for radiology procedures to confirm the following: correct patient, correct procedures to be carried out, correct procedure site and side, informed consent verification, and available implants and equipment functionality in this phase. Frequencies of contrast-medium extravasation and the resultant volume-dependent or 
severity-dependent allergy were investigated along with follow-up reviews of these incidents. Image quality assessment to determine diagnostic quality was studied in and compared between medical centres and local institutions to elucidate causes of rejected images (waste films resulting in additional exposure) and suboptimal radiographs (suboptimal quality but interpretable image) as well as the retake rates (repeated imaging due to rejected images). Finally, patient satisfaction with CT, MRI, mammography, ultrasonography and special procedures was assessed from responses to the questionnaires.

\section{Evaluations of the postexamination phase}

Error rates in radiology reports were determined by revision rates, and the reasons and conditions for making these corrections were studied. The report turnaround time of different examinations was evaluated for different types of patients, for example, inpatients, outpatients and emergency medical services (EMS) patients. Response rates of high-risk reminders for effective risk communication were surveyed. Postincident reviews and management as well as analyses of patients' appeals were compared between large medical centres and local institutions.

\section{Descriptive survey}

The 12 surveyed themes describing the status of patient safety were ranked in the order of importance.

\section{Statistical analysis}

Survey responses were collected for statistical analysis while the missing data were evaluated for quality assurance. The two-sample t-test and Mann-Whitney $\mathrm{U}$ test were used to determine the correlation between numeric and ordinal variables, respectively, while the Pearson $\chi^{2}$ test was used to determine the correlation between nominal variables at a statistical significance level of $\mathrm{p} \leq 0.050$.

\section{RESULTS}

Survey response rates, regardless of the missing data, were $95 \%$ and $100 \%$ for medical centres and local institutions, respectively. 'Patient verification', 'the records of contrast-medium extravasation and allergic reactions' and 'the establishment of high-risk reminder reporting systems' were selected by the medical centres as the most important means of ensuring or improving patient safety, whereas 'patient falls' and 'patient consents for examinations' instead of 'the records of contrastmedium extravasation and allergic reactions' were the selected items of local institutions.

The most important safety issue in the Department of Radiology was the correctness of patient identification and status. The number of credit hours of patient safety training or introduction of new employees or medical practitioners to their jobs in diagnostic radiology was similar in medical centres and local institutions.

\section{Results of the pre-examination phase evaluation}

Appropriateness of examination orders and practice

The inclusion of a clinical history in the examination orders was more commonly performed in medical centres than that in local institutions (see online supplementary table S1). The percentage of clinicians who included a complete medical history with the examination order $<40 \%$ of the time was smaller at medical centres than that at local institutions, that is, $11 \%$ vs $53 \%(\mathrm{p}=0.009)$, whereas the percentage of clinicians who included a complete clinical history $60 \%$ or more of the time was significantly larger in medical centres than that in local institutions ( $72 \%$ vs $34 \%$; $\mathrm{p}=0.026$ ).

\section{Access and wait times for radiological examinations}

The average times to access radiological examinations, including MRI, CT, ultrasonography, mammography and special procedures, were longer in medical centres than those in local institutions. In particular, the times (for medical centres vs local institutions) to the next available MRI were $7.00 \pm 29.50$ vs $3.50 \pm 3.50$ days $(\mathrm{p}=0.034)$ (see online supplementary table S2). In addition, the wait time for general radiographies was similar between medical centres and local institutions.

\section{Healthcare education}

In medical and local institutions, patient education was conducted prior to each examination. The percentage of patient education sessions led by radiologists or radiology residents (medical centres vs local institutions, $34 \%$ vs $17 \%$; $\mathrm{p}=0.014$ ) and/or radiologic technologists (medical centres vs local institutions, $65 \%$ vs 97\%; $\mathrm{p}=0.008$ ) was significantly different between medical and local institutions. A significantly higher percentage of patient education for CT (medical centres vs local institutions, $28 \%$ vs $6 \% ; \mathrm{p}=0.028)$ and special procedures (medical centres vs local institutions, $78 \%$ vs 6\%; $\mathrm{p}=0.042$ ) was carried out by radiologists or radiology residents in medical centres than that in local institutions (see online supplementary table S3). In contrast, a significantly higher percentage of patient education for MRI (medical centres vs local institutions, $56 \%$ vs $88 \%$; $\mathrm{p}=0.041)$ was carried out by radiologic technologists in local institutions.

\section{Examination order-based selection of imaging protocols}

At medical centres, standardised imaging protocols based on current standards of practice were used for all examinations, except ultrasonography (see online supplementary table S4). At local institutions, standardised imaging protocols for special procedures were significantly less used (medical centres vs local institutions, $100 \%$ vs $75 \%$; $\mathrm{p}=0.024)$. 
Results of the midexamination phase evaluation

Patient verification, time-out procedure and fall analyses

There were no significant differences between the medical centres and local institutions with respect to verification of patient identity and pregnancy status prior to radiological examinations. The rate of time-out procedure implementation for checking patients' consents and identities, invasive procedures, puncture sites/ sides as well as the readiness of instruments was indistinguishable in $>80 \%$ of medical and local institutions (see online supplementary table S5). The percentage of patient falls investigated was higher in medical centres than that in local institutions ( $100 \%$ vs $47 \%$, respectively; $\mathrm{p}=0.024)$ (table 1).

\section{Evaluation of side effects from intravenous contrast-medium administration}

Medical and local institutions kept records of contrast-medium extravasation and the resultant allergic reaction (table 2). Review and follow-up of such incidents and attempts at improvement, however, were significantly less frequent in local institutions (medical centres vs local institutions, $100 \%$ vs $77 \%$; $\mathrm{p}=0.029$ ). The records showed that contrast-medium extravasation at volumes larger than $20 \mathrm{~cm}^{3}$ occurred more frequently in medical centres $(2.75 \pm 1.00$ vs $1.00 \pm 0.75$ cases/year, $\mathrm{p}=0.050$; table 2) than that in local institutions.

\section{Assessment of image quality}

Rejected films and suboptimal radiographs were analysed and image retakes were monitored in $>60 \%$ of medical centres and local institutions. The frequency of

Table 1 Status of patient falls in medical centres versus local institutions

\begin{tabular}{|c|c|c|}
\hline Place of occurrence & $\begin{array}{l}\text { No. of } \\
\text { hospitals (\%) }\end{array}$ & p Value \\
\hline \multicolumn{3}{|c|}{ Records of patient falls } \\
\hline \multicolumn{3}{|c|}{ Standing radiography room } \\
\hline $\mathrm{MC}(\mathrm{N}=18)$ & $13(72)$ & 0.824 \\
\hline LI $(\mathrm{N}=16)$ & $11(69)$ & \\
\hline \multicolumn{3}{|l|}{ Dressing room } \\
\hline$M C(N=18)$ & $0(0)$ & 1.000 \\
\hline $\mathrm{LI}(\mathrm{N}=16)$ & $0(0)$ & \\
\hline \multicolumn{3}{|l|}{ Others* } \\
\hline $\mathrm{MC}(\mathrm{N}=18)$ & $5(28)$ & 0.545 \\
\hline LI $(\mathrm{N}=16)$ & $6(38)$ & \\
\hline \multicolumn{3}{|l|}{ Review of patient falls } \\
\hline \multicolumn{3}{|c|}{ Performed investigation $\dagger$} \\
\hline $\mathrm{MC}(\mathrm{N}=18)$ & $18(100)$ & 0.000 \\
\hline $\mathrm{LI}(\mathrm{N}=17)$ & $8(47)$ & \\
\hline \multicolumn{3}{|c|}{$\begin{array}{l}\text { The } \chi^{2} \text { test was applied for the statistical analysis. } \\
\text { *Others include the waiting areas in medical centres and the } \\
\text { return pathways as well as the examination tables and rooms, and } \\
\text { the corridors in local institutions. } \\
\text { †Significant statistical difference }(p<0.050) \text { in the performance of } \\
\text { patient fall investigations between medical and local institutions. } \\
\text { LI, local institutions; MC, medical centres. }\end{array}$} \\
\hline
\end{tabular}

suboptimal radiographs was significantly greater in local institutions than that in medical centres $(0.64 \pm 1.84 \%$ vs $0.00 \pm 0.01 \%$ per month, $\mathrm{p}=0.001)$, while the retake rate was significantly lower in medical centres $(0.03$ $\pm 0.04 \%$ vs $1.00 \pm 2.18 \%$ per month, $\mathrm{p}=0.029$; see online supplementary table $\mathrm{S} 6$ ). The two major causes of image rejection were 'incorrect positioning' and 'wrong imaging location.' Blank images contributed to the failure of imaging more frequently in medical centres than those in local institutes ( $41 \%$ vs $8 \%, \mathrm{p}=0.004)$.

\section{Surveys for patient satisfaction}

The rate of patient satisfaction with individual examinations was similar between medical centres and local institutions, but the rate of patient satisfaction with examinations overall was significantly higher in medical centres $(74 \%$ vs $57 \%, \quad \mathrm{p}=0.016 ;$ see online supplementary table $\mathrm{S} 7$ ).

\section{Results of the postexamination phase evaluation}

Correction of examination results

Revision of examination results by radiologists was more frequent in medical centres $(67 \%$ vs $24 \% ; \mathrm{p}=0.010)$ than that in local institutions during 2014 (table 3). In

Table 2 Incidents of extravasation of contrast media and contrast medium-induced allergy in medical centres and local institutions (unit: cases per year)

\begin{tabular}{lccc}
\hline & Median & IQR & p Value \\
\hline $\begin{array}{l}\text { Frequency of contrast-medium } \\
\text { Large volume* }\end{array}$ & & & \\
MC (N=16) & 2.75 & 1.00 & 0.050 \\
LI $(\mathrm{N}=16)$ & 1.00 & 0.75 & \\
Small volume & & & \\
MC (N=15) & 5.00 & 1.51 & 0.342 \\
LI $(\mathrm{N}=16)$ & 6.00 & 3.00 &
\end{tabular}

Frequency of allergic reaction to contrast media Severe casest

\begin{tabular}{lrrr} 
MC $(\mathrm{N}=14)$ & 0.25 & 0.88 & 0.246 \\
LI $(\mathrm{N}=16)$ & 0.00 & 0.13 & \\
Mild cases & & & \\
MC $(\mathrm{N}=17)$ & 12.00 & 30.00 & 0.728 \\
LI $(\mathrm{N}=16)$ & 20.00 & 23.25 & \\
\hline
\end{tabular}

\begin{tabular}{|c|c|c|c|}
\hline & No. of hospitals & Per cent & p Value \\
\hline \multicolumn{4}{|c|}{ Review and improvements } \\
\hline \multicolumn{4}{|c|}{ Cases of allergic reactions $\ddagger$} \\
\hline $\mathrm{MC}(\mathrm{N}=18)$ & 18 & 100 & 0.029 \\
\hline $\mathrm{LI}(\mathrm{N}=17)$ & 13 & 77 & \\
\hline
\end{tabular}

The Mann-Whitney $U$ test was used to analyse contrast extravasation and allergic reactions; an IQR was acquired from subtracting the 25 th centile from the 75 th centile. The $\chi^{2}$ test was used to analyse the review and improvements.

*Significant statistical difference $(p=0.050)$ in the frequency of large-volume (volume $>20 \mathrm{~cm}^{3}$ ) contrast extravasation between medical centres and local institutions.

tSevere allergic reactions include coma and death. $¥$ Significant statistical difference $(p<0.050)$ in the rates of reviewing allergic reaction to contrast agent incidents and improvement between medical centres and local institutions. $\mathrm{LI}$, local institutions; MC, medical centres. 
Table 3 Comparison of the rates of report revision by radiologists in medical centres versus local institutions

\section{No. of hospitals Per cent p Value}

Analysis and evaluation of the revision rate of examination reports*

$\begin{array}{lrrr}\text { MC }(\mathrm{N}=18) & 12 & 67 & 0.010 \\ \mathrm{LI}(\mathrm{N}=17) & 4 & 24 & \end{array}$

Major causes for report revision

Correction made after discussion with clinician

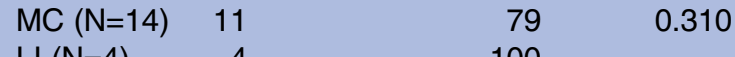

LI $(\mathrm{N}=4) \quad 4 \quad 100$

Correction made after comparing present with preceding imagest
$\mathrm{MC}(\mathrm{N}=14)$
29
0.011

$\mathrm{LI}(\mathrm{N}=4)$

Correction made at the clinician's request

$\mathrm{MC}(\mathrm{N}=14) \quad 9 \quad 64$

$\mathrm{LI}(\mathrm{N}=4) \quad 3 \quad 75$

After consideration of other examination result(s)

\begin{tabular}{llll} 
MC $(\mathrm{N}=14)$ & 9 & 64 & 0.689 \\
LI $(\mathrm{N}=4)$ & 3 & 75 & \\
$\begin{array}{l}\text { Perception errors } \\
\text { MC }(\mathrm{N}=14)\end{array}$ & 9 & & \\
$\mathrm{LI}(\mathrm{N}=4)$ & 3 & 64 & 0.689 \\
\hline
\end{tabular}

The $\chi^{2}$ test was used for statistical analysis.

*Significant statistical difference $(p<0.050)$ in the rate of analysis and evaluation of examination report corrections between medical and local institutions.

†Significant statistical difference $(p<0.050)$ in the rate of corrections made after comparing present with past images between medical and local institutions.

$\mathrm{LI}$, local institutions; MC, medical centres.

medical centres, corrections were more frequently made after discussion with clinicians, at the clinician's request, after consideration of other examination result(s), or after finding perception errors. In local institutions, they were more frequently made after comparing the new images with older ones (medical centres vs local institutions, $29 \%$ vs $100 \%$; $\mathrm{p}=0.011)$.

\section{Turnaround times for reporting examination results}

The report turnaround times for individual radiological examinations were similar for inpatients, EMS patients, outpatients and across centres (see online supplementary table S8). In general, the average report turnaround time was shorter for EMS patients than inpatients or outpatients. Specifically, the average turnaround time for special procedure reports was significantly shorter for EMS patients than inpatients in medical centres $(0.70 \pm 0.41$ vs $2.21 \pm 0.85$ days; $\mathrm{p}<0.001)$ but not local institutions ( $1.25 \pm 1.67$ vs $2.31 \pm 1.74$ days; $\mathrm{p}=0.095)$.

\section{High-risk incident reporting}

The high-risk incidents, including infections, occult bone fracture, internal haemorrhage, unexpected tumours, pneumothorax and tuberculosis, were reported immediately to associated personnel or departments through emails or phone calls. Even though all medical centres and local institutions did establish reporting high-risk reminder systems for high-risk incidents, the reporting rate was higher at local institutions than that at medical centres (table 4).

\section{Incident events and the review conferences}

The yearly average number of incident events was markedly smaller at medical centres than that at local institutions (see online supplementary table S9). Further, a significantly lower percentage of local institutions carried out regular incident review conferences $(89 \%$ vs $47 \% ; \mathrm{p}=0.008$ ).

\section{DISCUSSION}

Under the constraints of examination time and workforce supply, ${ }^{16}$ patient safety during radiology procedures in Taiwan is threatened by medical errors and examination-related adverse events and incidents. ${ }^{15}$ The status of patient safety surveyed in medical centres and local institutions reflects differences in quality standards of care in diagnostic radiology departments.

\section{Pre-examination phase}

Well-established referral mechanism in the medical centres

Radiologists did not routinely review requests for CT and MRI before carrying out these examinations in some of the medical centres and local institutions surveyed; therefore, leaving the appropriateness of examination orders to be decided by radiologic technologists may easily lead to misunderstandings. The practice of including a complete medical history with the examination orders was particularly prevalent at medical centres and may have played a crucial role in ensuring the appropriateness of examination orders to meet the standard of care.

\section{Increased performance efficiency}

The level of examination order appropriateness was higher and the average times to access MRI were longer at medical centres, despite having greater numbers of hospital beds and the most expensive medical devices. The longer access times reflect the high demand for medical imaging services and indicate a potential deficiency in the workforce and/or excessive imaging

Table 4 Comparison of the rates of high-risk incident reporting between medical centres and local institutions (unit: monthly percentage)

\begin{tabular}{llll}
\hline & Median & IQR & p Value \\
\hline $\mathrm{MC}(\mathrm{N}=9)$ & 0.01 & 0.16 & 0.091 \\
$\mathrm{LI}(\mathrm{N}=7)$ & 1.00 & 1.75 & \\
\hline
\end{tabular}

The Mann-Whitney $U$ test was used for the statistical analysis. An IQR was acquired from subtracting the 25th centile from the 75th centile of a set of non-normal distributed data.

$\mathrm{LI}$, local institutions; MC, medical centres. 
instrument workloads. In such a situation, the efficiency of hospital work is inevitably impaired and quality of care reduced. The quality of patient care and services can be optimised by access time policies that favour EMS patients over inpatients and outpatients. In fact, a significantly high percentage of medical centres have their radiologists and radiology residents rather than radiologic technologists participate in patient education. Such a strategy promotes patient safety while reducing examination time.

\section{Patient safety enhancement in the medical Centres}

Greater use of standardised imaging protocols should increase patient safety while performing special procedures. Notably, the use of standardised imaging protocols for different subgroups such as adults/children, body areas and projections can produce excellent radiographs and reduce the number of and need for repeated examinations. ${ }^{17}$ For special radiological procedures, standardised operative procedures with condition-dependent optimisation can reduce examination complications and time. ${ }^{18}$ Therefore, a large percentage of medical centres provide a secure environment for the patients and participating medical staff by following imaging protocols for use in interventional procedures. As dose minimisation is a major aspect of patient safety in diagnostic radiology, ${ }^{19}$ strategies to minimise radiation dose (ie, correct selection and implementation of imaging protocols) are extremely important. ${ }^{14}{ }^{17}$ Medical centres require strict policies and strategic measures to improve work efficiency while assuring patient safety and preventing medical malpractice.

\section{Midexamination phase}

\section{Safety control in the medical centres}

Patient falls and incidents of contrast-induced allergic reaction were recorded and investigated at medical centres. Presumably, preventive measures for falls and fall-prevention accessories (eg, safety cushioning back belt) for elderly patients who undergo standing radiography or examinations that require patients to sit or lay on examination tables were especially emphasised at medical centres, because they constituted the second leading cause of accidental death in elderly people aged 65 or older. ${ }^{13}$ Moreover, the risk of extravasation of a large amount of contrast agent through power injection is increased in medical centres, as they treat patients who are more severely ill or injured. ${ }^{20} 21$ Preventive measures for avoiding the extravasation of contrast materials include decreasing the injector pressure, ensuring the return and circulation of venous blood, careful choice of intravenous administration site and close monitoring of patients during injection of contrast agents. ${ }^{22}{ }^{23}$ In spite of the difference in standards of care in diagnostic radiology settings between medical centres and local institutions, specialised training in patient safety and patient education in contrast agent administration or examination positioning does not clearly recommend ways to avoid contrast agent extravasation or patient falls, respectively. ${ }^{23} 24$

\section{Image quality assurance: needed for improving workforce} skills

Image retake rates at medical centres and local institutions were similar, and the major cause of image rejection at both centres was incorrect patient positioning. Improper positioning could lead to overcollimation (inappropriate use of small radiation fields) and thereby to overexposure when applying an automatic exposure control system. ${ }^{17} 25$ In contrast, the significantly higher rate of blank images at medical centres, although not contributing to radiation exposure of patients, may be associated with inappropriate use of large radiation fields due to wrong positioning and inappropriate collimating, not to mention that operator errors in the two-step exposure process can also result in wrong determination of $\mathrm{kVp}$ and $\mathrm{mA}$ outputs and the subsequent underexposure of radiographs or detectors. ${ }^{17}{ }^{25}$ Sufficient imaging procedure training of future and present technologists holds the key to improvement of patient safety.

\section{Patient experiences and feedback}

A larger number of medical centres than local institutions conducted surveys on patient satisfaction every year, indicating their greater level of commitment to continuous improvement and excellence in patient care in diagnostic radiology. The practices of nursing staff and radiologic technologists are as critically important to patient safety as those of radiologists.

\section{Postexamination phase}

\section{Correctness of examination reports}

Differences in the correctness of examination reports and retrospective review of reports between medical centres and local institutions attest to differences in operational standards and competencies of medical care providers in diagnostic radiology. Revision of examination reports made after comparing present with preceding images was significantly more common in local institutions, while correction after reviewing reports was significantly more common in medical centres, thereby implying the greater effectiveness of examinations reported by radiologists at medical centres.

\section{Hospital system efficiency: effective workflow management and short report turnaround times}

Considering that the turnaround time of examination reports strongly affects the patient's wait time for subsequent treatment and management, factors that affect report delivery time such as the amount and allocation of facilities, workload and complexity of diseases need to be considered when optimising procedures and improving correctness and efficiency. ${ }^{26}$ The turnaround time for special procedure radiology reports was significantly shorter at medical centres for EMS patients than inpatients. This result highlights the fact that radiology 
departments at medical centres expedite the processing and management of emergency and critical cases.

\section{Potential misuse of high-risk reminder systems in local institutions}

Reporting effectiveness was also evaluated by rate estimates of high-risk incidents. The establishment of a high-risk reminder reporting system has shortened the delivery time of critical healthcare to patients with highrisk symptoms. ${ }^{27}$ Text messages can be quickly sent from radiologists to clinicians upon detection of high-risk symptoms, and immediate risk management actions can be taken in response. ${ }^{28}$ However, excessive and nonessential reporting of risk can likely compromise the efficacy of risk management in high-risk patients. Local institutions, with a median reporting rate of $1 \%$, provided unnecessary, extraneous care to some patients may be misclassified as high-risk patients. ${ }^{13}$ Therefore, effective management of examination results and diagnostic imaging reports at local institutions is essential to mitigate or redefine risk and thereby improve patient safety.

\section{Summary}

This survey discusses the present status of patient safety in all phases of diagnostic radiology processes and for all operational procedures, including preoperational education, prospective monitoring and retrospective incident review. In view of the varied nationwide patient care in diagnostic radiology, we would recommend local institutions to implement more complete pre-examination procedures such as the use of imaging protocols and inclusion of medical history in examination orders. In addition, it is recommended to conduct site visits and staff interviews between medical centres and local institutions to validate some of the identified events such as the significantly higher rates of contrast extravasation and blank images and relatively high reporting rate of high-risk incidents. With constant technological advancement, continuous personnel training on patient administration, imaging and diagnosis is critical to improve the quality of care and reduce patient harm. The results of our survey may shed light on the future development of safety measures and management in medical centres and local institutions, including data-driven adaptive control approaches to improve standards of medical service delivery in diagnostic radiology.

\author{
Author affiliations \\ ${ }^{1}$ Department of Radiology, Wan Fang Hospital, Taipei Medical University, \\ Taipei, Taiwan \\ ${ }^{2}$ Department of Radiology, Taichung Veterans General Hospital, Taichung, \\ Taiwan \\ ${ }^{3}$ Department of Radiology, Taipei Medical University Hospital, Taipei, Taiwan \\ ${ }^{4}$ Department of Radiology, School of Medicine, College of Medicine, Taipei \\ Medical University, Taipei, Taiwan \\ ${ }^{5}$ Institute for Radiological Research, College of Medicine, Chang Gung \\ University, Taoyuan City, Taiwan \\ ${ }^{6}$ Department of Radiology, Taipei Veterans General Hospital, Taipei, Taiwan
}

Acknowledgements The authors thank all the individuals at the medical centres and local institutions for participating in the survey. The authors also thank Dr Yan-Guang Lin for his assistance with statistical analyses.

Contributors WPC contributed to conception and design. Y-HL, CC-CC, S-KL and WPC developed the methodology. CC-CC, C-YC, Y-LW, W-YG, AC and WPC contributed to acquisition of data. Y-HL, CC-CC, AC and WPC carried out the analysis and interpretation of data. Y-HL wrote the manuscript. Y-HL, CC-CC, S-KL, W-YG and WPC contributed to study supervision. All authors have reviewed and revised the manuscript for important intellectual content. All authors have approved the version to be published.

Competing interests None declared.

Provenance and peer review Not commissioned; externally peer reviewed.

Data sharing statement No additional data are available.

Open Access This is an Open Access article distributed in accordance with the Creative Commons Attribution Non Commercial (CC BY-NC 4.0) license, which permits others to distribute, remix, adapt, build upon this work noncommercially, and license their derivative works on different terms, provided the original work is properly cited and the use is non-commercial. See: http:// creativecommons.org/licenses/by-nc/4.0/

\section{REFERENCES}

1. Mello MM, Hemenway D. Medical malpractice as an epidemiological problem. Soc Sci Med 2004;59:39-46.

2. Ko Y, Yu S. The relationships among perceived patients' safety culture, intention to report errors, and leader coaching behavior of nurses in Korea: a pilot study. J Patient Saf 2015.

3. Hiivala N, Mussalo-Rauhamaa $\mathrm{H}$, Tefke $\mathrm{HL}$, et al. An analysis of dental patient safety incidents in a patient complaint and healthcare supervisory database in Finland. Acta Odontol Scand 2016;74:81-9.

4. Lee $\mathrm{CH}$, Lin MF, Chan WP. Management of a sandbag accident in an MRI unit. Magn Reson Imaging 2015;33:1187-9.

5. House of Commons (Parliament, Great Britain). Commissioning: written evidence. London: The Stationery Office, 2010.

6. Sage WM, Jablonski JS, Thomas EJ. Use of nondisclosure agreements in medical malpractice settlements by a large academic health care system. JAMA Intern Med 2015;175:1130-5.

7. http://www.aec.gov.tw/webpage/service/download/append_09-1_12. pdf (accessed 7 Oct 2015).

8. http://www.aec.gov.tw/webpage/service/download/append_09_09.pdf (accessed 7 Oct 2015).

9. http://www.aec.gov.tw/webpage/service/files/seminar_2007-8.pdf (accessed 7 Oct 2015).

10. http://acs.ait.org.tw/medical-info.html (accessed 1 Sep 2015)

11. http://psi.tmu.edu.tw/\%E7\%97\%85\%E4\%BA\%BA\%E5\%AE\%89\% E5\%85\%A8\%E6\%89\%8B\%E5\%86\%8A94.pdf (accessed 1 Sep 2015).

12. http://www.tjcha.org.tw/FrontStage/page.aspx?ID=FE5F1FDE-28394702-BA2A-A9DE1D3672FB\&PID=637D42A4-9D35-407A-B01E2EB3CAE2FOD7 (accessed 10 Oct 2015).

13. Chan WP, Lai YJ, Chen CY, et al. Survey of patient safety in the examination process of diagnostic radiology departments at medical centers in Taiwan. Chin J Radiol 2007;32:193-202.

14. Johnson CD, Krecke KN, Miranda R, et al. Quality initiatives: developing a radiology quality and safety program: a primer. Radiographics 2009;29:951-9.

15. Chang PY, Hsieh MC, La YJ, et al. Analysis of revised imaging reports in diagnostic radiology: an example from a medical center in Taiwan. Chin J Radiol 2008;33:23-9.

16. Chan WP, Hung ST, Chang CY, et al. Supply and demand in relation to diagnostic radiologists in Taiwan over the next 10 years. Chin J Radiol 2007;32:71-80.

17. Long BW, Frank ED, Ehrlich RA. Radiography essentials for limited practice. St. Louis (MO): Elsevier Health Sciences, 2014:63-76.

18. Snopek AM. Fundamentals of special radiographic procedures. St. Louis (MO): Elsevier Health Sciences, 2013:201-431.

19. Amis ES, Butler PF, Applegate KE, et al. American College of Radiology White paper on radiation dose in medicine. J Am Coll Radiol 2007;4:272-84.

20. Shaqdan K, Aran S, Thrall J, et al. Incidence of contrast medium extravasation for CT and MRI in a large academic medical centre: a report on 502,391 injections. Clin Radiol 2014;69:1264-72.

21. ACR Committee on Drug and Contrast Media. Extravasation of contrast media. ACR Manual on Contrast Media, 2012:17-19. http:// 
radres.ucsd.edu/documents/Body/Extravasation\%20of\%20Contrast \%20Media.pdf (accessed 23 Sep 2015).

22. Belzunegui T, Louis CJ, Torrededia L, et al. Extravasation of radiographic contrast material and compartment syndrome in the hand: a case report. Scand J Trauma Resusc Emerg Med 2011;19:9.

23. Morrison G, Odle TG. Safety considerations in contrast media handling and administration. Am Soc Radiol Technol 2007. http://www.asit.org/docs/default-source/whitepapers/ km07whtpprcontrastsafetyfinal2081307.pdf?sfvrsn=2 (accessed 22 Sep 2015).

24. Abujudeh $\mathrm{H}$, Kaewlai $\mathrm{R}$, Shah $\mathrm{B}$, et al. Characteristics of falls in a large academic radiology department: occurrence, associated factors, outcomes, and quality improvement strategies. $A m$ $J$ Roentgenol 2011;197:154-9.

25. Johnson J, Fauber TL. Essentials of radiographic physics and imaging. St. Louis (MO): Elsevier Health Sciences, 2013:175-84.

26. Chiang YC, Hsieh YS, Chao SH, et al. A study on quality improvement of delivering examination reports at the radiology department. J Healthc Manag 2002;3:41-60.

27. Aragones A, Bruno DM, Ehrenberg M, et al. Parental education and text messaging reminders as effective community based tools to increase HPV vaccination rates among Mexican American children. Prev Med Rep 2015;2:554-8.

28. Dimitris K. Services customization using web technologies. Hershey (PA): Business Science Reference, 2012:171-86. 


\section{Correction}

Lee Y-H, Chen CC-C, Lee S-K, et al. Patient safety during radiological examinations: a nationwide survey of residency training hospitals in Taiwan. BMJ Open 2016;6: e010756. doi:10.1136/bmjopen-2015-010756

In the Abstract, the text "medical centres had lower monthly rates of suboptimal (but interpretable) radiographs $(0.00 \pm 0.01 \%$ vs. $0.64 \pm 1.84 \%)$ " should read "medical centres had an overall insignificantly lower monthly rate of suboptimal (but interpretable) radiographs than local institutions $(0.40 \pm 4.10 \%$ vs. $2.00 \pm 5.23 \%)$.

In Supplemental Table S6 and the Results section. In (Midexamination phase evaluation) the paragraph entitled "Assessment of image quality" in the Results, the first sentence, "Rejected films and suboptimal radiographs were ..... in $>60 \%$ of medical centres and local institutions," should have read "Rejected films and suboptimal radiographs were ..... in $<50 \%$ of medical centres and local institutions". The second sentence should say: "The frequency of suboptimal radiographs was not significantly different between medical centres and local institutions $(0.40 \pm 4.10 \%$ vs. $2.00 \pm 5.23 \%$ per month, $\mathrm{p}=0.549$ ), while the variation in retake rate was significantly smaller in medical centres $(3.00 \pm 1.00 \%$ vs. $2.00 \pm 16.37 \%$ per month, $\mathrm{p}=0.028)$ ". Finally, in the Image quality assurance: needed for improving workforce skills of the Discussion section, the first sentence, "Image retake rates at medical centres and local institutions ..... at both centres was incorrect patient positioning," should read "Even though image retake rates at medical centers and local institutions were significantly different, in both cases the image rejections were mainly attributed to incorrect patient positioning".

Here are the corrected numbers for the upper part of Supplementary Table 6:

\begin{tabular}{|c|c|c|c|}
\hline \multicolumn{4}{|c|}{ Frequencies of Defective Radiographs and Radiograph Retake } \\
\hline & \multicolumn{2}{|c|}{ (Unit: \% in a Month) } & \multirow[b]{2}{*}{ p-value } \\
\hline & Median & Interquartile Range & \\
\hline \multicolumn{4}{|c|}{ Retake Rate* } \\
\hline$M C(N=10)$ & 3.00 & 1.00 & 0.028 \\
\hline LI $(\mathrm{N}=7)$ & 2.00 & 16.37 & \\
\hline \multicolumn{4}{|c|}{$\begin{array}{l}\text { Rate of Suboptimal } \\
\text { Radiographs }\end{array}$} \\
\hline$M C(N=9)$ & 0.40 & 4.10 & 0.549 \\
\hline 니 $(N=5)$ & 2.00 & 5.23 & \\
\hline
\end{tabular}

Open Access This is an Open Access article distributed in accordance with the Creative Commons Attribution Non Commercial (CC BY-NC 4.0) license, which permits others to distribute, remix, adapt, build upon this work noncommercially, and license their derivative works on different terms, provided the original work is properly cited and the use is non-commercial. See: http://creativecommons.org/licenses/by-nc/4.0/

BMJ Open 2016;6:e010756corr1. doi:10.1136/bmjopen-2015-010756corr1

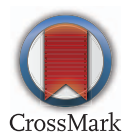

